\title{
A INFLUÊNCIA DAS HABILIDADES EM CONSCIÊNCIA FONOLÓGICA NA TERAPIA PARA OS DESVIOS FONOLÓGICOS
}

\author{
The influence of phonological awareness abilities \\ in therapy for phonological disorder
}

\author{
Carolina Lisbôa Mezzomo (1), Helena Bolli Mota ${ }^{(2)}$, Márcia Keske-Soares ${ }^{(3)}$, \\ Marizete Ilha Ceron ${ }^{(4)}$, Roberta Freitas Dias ${ }^{(5)}$
}

\begin{abstract}
RESUMO
Este trabalho teve como objetivo analisar as habilidades em consciência fonológica e o progresso (inventário fonético e fonológico e as generalizações) na terapia fonológica. O grupo pesquisado foi constituído por cinco crianças com desvio fonológico, com idades entre 5:0 e 6:11, submetidas à terapia fonológica. Foram analisados os resultados obtidos na avaliação da consciência fonológica pré-tratamento, a fim de verificar o desempenho das crianças quanto ao seu conhecimento fonológico. Realizou-se a avaliação fonológica pré e pós-tratamento, em que foi possível obter os inventários fonéticos e fonológicos dos sujeitos. Após, foram analisadas as generalizações obtidas com a terapia fonológica (itens lexicais não utilizados no tratamento, outras posições na palavra, dentro de uma classe de sons, outra classe de sons). Os resultados evidenciaram que não existe relação entre o desempenho em tarefas de consciência fonológica e os progressos na terapia. Tais resultados corroboram os achados da literatura, confirmando que crianças com desvio fonológico podem ser capazes de responder adequadamente a tarefas metalinguísticas como a consciência fonológica, sem que essa condição as auxilie a corrigir os desvios de sua fala. Sugere-se que este assunto seja mais bem investigado com um número maior de sujeitos, bem como com a avaliação da consciência fonológica pré e pós-terapia, no intuito de se obter dados generalizáveis os quais serão importantes para o entendimento dos casos de desvio fonológico.
\end{abstract}

DESCRITORES: Fala; Distúrbios da Fala; Percepção da Fala; Fonoterapia; Generalização da Resposta

(1) Fonoaudióloga; Professora Adjunta do Curso de Fonoaudiologia e do Programa de Pós-Graduação em Distúrbios da Comunicação Humana da Universidade Federal de Santa Maria - UFSM, Santa Maria-RS, Brasil; Doutora em Linguística Aplicada pela Pontifícia Universidade Católica do Rio Grande do Sul - PUCRS.

(2) Fonoaudióloga; Professora Associada do Curso de Fonoaudiologia e do Programa de Pós-Graduação em Distúrbios da Comunicação Humana da Universidade Federal de Santa Maria - UFSM, Santa Maria-RS, Brasil; Doutora em Linguística Aplicada pela Pontifícia Universidade Católica do Rio Grande do Sul - PUCRS.

(3) Fonoaudióloga; Professora Adjunta do Curso de Fonoaudiologia e do Programa de Pós-Graduação em Distúrbios da Comunicação Humana da Universidade Federal de Santa Maria - UFSM, Santa Maria-RS, Brasil; Doutora em Linguística Aplicada pela Pontifícia Universidade Católica do Rio Grande do Sul - PUCRS.

(4) Fonoaudióloga, Doutoranda em Distúrbios da Comunicação Humana pela Universidade Federal de Santa Maria -

\section{INTRODUÇÃO}

A evolução da habilidade de comunicação é favorecida pelo surgimento de processos de análise da fala, mais elaborados e lentos, denominados de consciência linguística. Esses processos envolvem a auto correção de erros durante as operações

UFSM, Santa Maria-RS, Brasil; Mestre em Distúrbios da Comunicação Humana pela Universidade Federal de Santa Maria - UFSM.

(5) Fonoaudióloga; Professora substituta do Curso de Fonoaudiologia da Universidade Federal de Santa Maria - UFSM e Doutoranda em Distúrbios da Comunicação Humana pela Universidade Federal de Santa Maria - UFSM, Santa Maria-RS, Brasil; Mestre em Distúrbios da Comunicação Humana pela Universidade Federal de Santa Maria UFSM.

Conflito de interesses: inexistente 
básicas de produção e compreensão da fala, a expansão e a testagem das possibilidades de utilização da língua durante a aquisição dessas habilidades comunicativas básicas, bem como a necessidade de aprender o código escrito'1.

A consciência fonológica é um subtipo de consciência linguística que se refere à habilidade de analisar a fala explicitamente em seus componentes fonológicos ${ }^{1}$. Ela parece se desenvolver gradativamente desde a consciência de unidades fonológicas mais globais - palavras e sílabas - até o desenvolvimento da consciência fonêmica. Os diferentes tipos de habilidades em consciência fonológica seguem, de maneira geral, uma sequência de emergência - habilidade em consciência de palavras, seguida pela habilidade em consciência de rimas, posteriormente de sílabas e, por último, de fonemas ${ }^{1}$.

As habilidades em consciência fonológica podem ou não estar defasadas nas crianças com desvios fonológicos quando comparadas às capacidades metalinguísticas de crianças com desenvolvimento fonológico típico.

A relação entre os desvios fonológicos e as dificuldades em consciência fonológica foi confirmada em pesquisas que observaram um desempenho inferior em provas de consciência fonológica por crianças com desvio ${ }^{2}$ em relação aquelas com desenvolvimento fonológico típico ${ }^{3-9}$. Por outro lado, há dados na literatura que evidenciaram que crianças com desvio fonológico não diferem no desempenho de tarefas de consciência fonológica quando comparadas a crianças com desenvolvimento típico ${ }^{10}$.

Crianças que apresentam alteração na organização mental dos fones contrastivos, em idades não mais esperadas, devem ser acompanhadas e tratadas. A terapia fonológica busca a adequação da fala pela reorganização do inventário fonológico, cuja função é a eficácia da comunicação ${ }^{11,} 12$.

Neste processo, o clínico tem como objetivo abreviar o tempo do início da terapia à alta, o que se obtém por meio da ocorrência da generalização, a qual é definida como a extensão ou a transferência do aprendizado. A generalização é a ocorrência dos sons tratados em outros contextos ou palavras não tratadas, podendo, ainda, ocorrer dentro de uma classe de sons ou para outras classes de sons ${ }^{11}$. Além da generalização, a análise pré e pós-tratamento dos inventários fonético e fonológico são úteis para medir as mudanças ocorridas com a terapia.

A terapia fonoaudiológica pode favorecer o desempenho em tarefas de consciência fonológica $^{7}$. Este aspecto foi demonstrado por meio dos resultados obtidos em um estudo que investigou o desempenho em consciência fonológica, de crianças com desvio fonológico, considerando a terapia fonoaudiológica. As crianças que receberam terapia fonológica, sem intervenção direta em consciência fonológica, obtiveram resultados melhores comparadas as crianças que não receberam tal intervenção? .

A partir disso, pode-se acreditar que crianças com um bom desempenho em tarefas de consciência fonológica apresentariam uma evolução mais rápida na terapia fonológica. Com isso, o objetivo deste relato de caso é analisar as habilidades em consciência fonológica e o progresso (inventário fonético e fonológico e as generalizações) na terapia fonológica.

\section{APRESENTAÇÃO DOS CASOS}

Este relato de caso está constituído por cinco crianças com desvio fonológico, com idades entre 5:0 e 6:11, sendo quatro do sexo masculino e um do sexo feminino. Os dados dos sujeitos selecionados para esta pesquisa fazem parte do Banco de Dados do Centro de Estudos de Linguagem e Fala de uma Instituição de Ensino Superior (IES), cujo projeto foi aprovado no Comitê de Ética em Pesquisa sob no 0103.0.243.000-07, da mesma IES. Os pais e/ ou responsáveis assinaram um Termo de Consentimento Livre e Esclarecido autorizando o uso dos dados referentes às avaliações e procedimentos de terapia em pesquisas e posterior publicação dos resultados em meio acadêmico, mantendo o anonimato.

Atualmente este banco de dados está composto por 193 sujeitos, os quais foram avaliados e submetidos a diferentes modelos de terapia fonológica (ABAB-Retirada e Provas Múltiplas, Ciclos Modificado, Pares Mínimos, Pares Mínimos/Oposições Máximas, Oposições Múltiplas). Para este estudo foram selecionados aleatoriamente os dados de cinco sujeitos, de acordo com os seguintes critérios de inclusão: diagnóstico de desvio fonológico; audição normal; não apresentar alterações nos aspectos neurológico, cognitivo e psicológico; ter os protocolos das avaliações da consciência fonológica (Protocolo de Tarefas de Consciência Fonológica proposto por Cielo ${ }^{1}$ ) e da fonologia completos; e ter sido submetido à terapia fonológica.

Todos os sujeitos participantes desta pesquisa passaram por uma triagem fonoaudiológica. Nesta triagem foram realizadas as seguintes avaliações: anamnese, linguagem compreensiva e expressiva, sistema estomatognático, exame articulatório, discriminação auditiva, consciência fonológica, processamento auditivo simplificado, vocabulário, avaliação audiológica e avaliação fonológica. Além 
disso, foram realizadas as avaliações complementares: otorrinolaringológica e neurológica, em que todos apresentaram resultados dentro dos padrões de normalidade. Com isso, pode-se confirmar o diagnóstico de desvio fonológico.

Após as avaliações, os sujeitos foram submetidos a diferentes abordagens de terapia fonológica. Essas abordagens de terapia foram escolhidas de acordo com as pesquisas que estavam sendo realizadas no CELF e conforme as alterações dos fonemas observadas no inventário fonológico pré-tratamento, os quais mostram as possibilidades de intervenção. Assim, S1, S2 e S3 foram submetidos à terapia pelo Modelo ABAB-Retirada e Provas Múltiplas ${ }^{13}$, em que foram analisados dois ciclos de terapia, aproximadamente dezoito sessões, com exceção o S1 que obteve alta da terapia com apenas nove sessões. O quarto (S4) e o quinto sujeito (S5) receberam tratamentos respectivamente pelos Modelos de Pares Mínimos ${ }^{14}$ e Pares Mínimos/Oposições Máximas ${ }^{15}$, nos quais se analisaram vinte cinco sessões de terapia. Conforme exposto acima, os sujeitos desta pesquisa receberam tratamento por modelos terapêuticos diferentes (ABAB-Retirada e Provas Múltiplas, Pares Mínimos, Pares Mínimos/Oposições Máximas), os quais não foram considerados para fim de análise em separado, pois há vários estudos ${ }^{16-19}$ que aplicaram esses modelos e apontam a eficácia deles no tratamento de crianças com desvio fonológico.

As avaliações que foram utilizadas para a análise nesta pesquisa são: consciência fonológica pré-tratamento e avaliações fonológicas pré e pós-tratamento dos cinco sujeitos. A avaliação da consciência fonológica teve como objetivo verificar o desempenho das crianças quanto ao seu conhecimento fonológico. Para a avaliação desta habilidade utilizou-se o Protocolo de Tarefas de Consciência Fonológica ${ }^{1}$. Este protocolo apresenta doze tarefas de consciência fonológica divididas em subtarefas respeitando uma ordem crescente de complexidade. As tarefas de consciência fonológica incluem segmentação de frases em palavras (T1); realismo nominal (T2); detecção de rimas (T3); síntese silábica (T4); segmentação silábica (T5); detecção de sílabas (T6); reversão silábica (T7); exclusão fonêmica (T8); detecção de fonemas (T9); síntese fonêmica (T10); segmentação fonêmica (T11) e reversão fonêmica (T12).

Os dados de fala foram obtidos pela aplicação do instrumento de avaliação fonológica por meio de nomeação espontânea de figuras temáticas, o que possibilitou a obtenção de uma amostra linguística significante com todos os fonemas da língua nas diferentes posições da palavra e em palavras diferentes. Esses dados foram gravados, transcritos foneticamente e analisados pela análise contrastiva, a qual permitiu obter os inventários fonéticos e fonológicos dos sujeitos. No inventário fonético considerou-se um som como presente, quando ocorreu, no mínimo, duas vezes em qualquer posição da palavra. No inventário fonológico considerou-se um fonema adquirido quando este ocorreu em $80 \%$ ou mais das possibilidades. Também, analisou-se o progresso na terapia pela ocorrência dos tipos de generalização (itens lexicais não utilizados no tratamento, outras posições na palavra, dentro de uma classe de sons, outra classe de sons).

\section{RESULTADOS}

Os sujeitos S1, S2 e S3 não tinham sons ausentes no seu inventário inicial para acrescentar, enquanto que os sujeitos S4 e S5 apresentavam vários $(S 4-[g, v, t, d, 3, R, d z, \kappa, r]$ e $S 5-[k, g, v, R$, $d 3, \kappa, r]$ ) sons ausentes no inventário fonético, dos quais todos foram incluídos, com exceção do [g] que permaneceu ausente para ambos os sujeitos. Os sujeitos S4 e S5 são os que apresentavam mais fonemas ausentes e parcialmente adquiridos no inventário fonológico pré e pós-terapia (Tabela 1). 
Tabela 1 - Sons ausentes no inventário fonético e fonemas parcialmente adquiridos e ausentes no inventário fonológico dos sujeitos

\begin{tabular}{|c|c|c|c|c|}
\hline \multirow{2}{*}{ Sujeitos } & & \multirow{2}{*}{$\begin{array}{c}\text { Inventário Fonético } \\
\text { SA }\end{array}$} & \multicolumn{2}{|c|}{ Inventário Fonológico } \\
\hline & & & FPA & FA \\
\hline \multirow{2}{*}{ S1 } & Al & - & - & $r$ \\
\hline & AF & - & - & - \\
\hline \multirow{2}{*}{$\mathbf{S 2}$} & Al & - & - & $\kappa, r$ \\
\hline & $\mathbf{A F}$ & - & $r$ & - \\
\hline \multirow{2}{*}{ S3 } & Al & - & - & $r$ \\
\hline & $\mathbf{A F}$ & - & - & $r$ \\
\hline \multirow[t]{2}{*}{ S4 } & Al & $\mathrm{g}, \mathrm{v}, \mathrm{t}, \mathrm{d}, 3, \mathrm{R}, \mathrm{d}_{3}, \kappa, \mathrm{r}$ & - & $\begin{array}{c}\mathrm{b}, \mathrm{d}, \mathrm{k}, \mathrm{g}, \mathrm{v}, \mathrm{s}, \mathrm{z} \\
\int, 3, \mathrm{l}, \kappa, \mathrm{r}, \mathrm{R}\end{array}$ \\
\hline & $\mathbf{A F}$ & g & $b, d, s, \kappa$ & $\mathrm{k}, \mathrm{g}, \mathrm{v}, \mathrm{z}, \mathrm{z}, \mathrm{l}, \mathrm{\Lambda}, \mathrm{r}, \mathrm{R}$ \\
\hline \multirow{2}{*}{ S5 } & Al & $\mathrm{k}, \mathrm{g}, \mathrm{v}, \mathrm{R}, \mathrm{d}_{3}, \kappa, \mathrm{r}$ & $d, z$ & $\mathrm{~b}, \mathrm{k}, \mathrm{g}, \mathrm{v}, 3, \mathrm{l}, \kappa, \mathrm{r}, \mathrm{R}$ \\
\hline & $\mathbf{A F}$ & $\mathrm{g}$ & $b, d, v, z, 3, R$ & $\mathrm{k}, \mathrm{g}, \mathrm{l}, \mathrm{r}, \mathrm{\kappa}$ \\
\hline
\end{tabular}

Legenda: Al: avaliação inicial; AF: avaliação final; SA: sons ausentes; FPA: fonema parcialmente adquirido; FA: fonema ausente.

Observa-se o diferencial entre o pré e pós-tratamento no que se refere ao número de sons adquiridos no inventário fonético, número de fonemas estabelecidos no inventário fonológico e o percentual de ocorrência de generalizações (a itens não utilizados no tratamento, para outra posição na palavra, para outra classe de sons e dentro de uma classe de sons) nos cinco sujeitos estudados (Tabela 2).

O S1 foi o sujeito que obteve os melhores resultados, havendo $100 \%$ de generalização a itens lexicais não utilizados no tratamento e para outra posição na palavra. S1, S2 e S3 não poderiam apresentar generalização dentro de uma classe de sons e para outra classe de sons devido aos seus inventários fonológicos não apresentarem outras alterações de fonemas. Da mesma forma, o S5 não poderia generalizar para outra classe de sons, pois todas as classes de sons que apresentavam alterações foram tratadas na terapia. O S4 apresentou todos os tipos de generalizações, sendo os maiores percentuais obtidos para a generalização dentro de uma classe de sons e para itens lexicais não utilizados no tratamento.

Em geral os sujeitos S1, S3 e S4 foram os que obtiveram um melhor resultado quanto ao desempenho nas tarefas de consciência fonológica (Figura 1).

Tabela 2 - Inventários fonético e fonológico pré tratamento e a ocorrência de generalizações após a terapia fonológica nos cinco sujeitos estudados

\begin{tabular}{|c|c|c|c|c|c|c|}
\hline \multirow{2}{*}{ Sujeitos } & \multirow{2}{*}{ IFe } & \multirow{2}{*}{ IFo } & \multicolumn{4}{|c|}{ Generalizações } \\
\hline & & & INUT (\%) & OPP (\%) & DCS (\%) & OCS (\%) \\
\hline S1 & 1 & 1 & 100 & 100 & - & - \\
\hline S2 & 1 & 0 & 8 & 2,5 & - & - \\
\hline s3 & 0 & 0 & 14,6 & 11,58 & - & - \\
\hline S4 & 8 & 0 & 44,82 & 30,48 & 51,35 & 28,88 \\
\hline S5 & 6 & 1 & 28,41 & 52,75 & 28,44 & - \\
\hline
\end{tabular}

Legenda: IFe: inventário fonético; IFo: inventário fonológico; INUT: generalização a itens lexicais não utilizados no tratamento; OPP: outras posições na palavra; DCS: dentro de uma classe de sons; OCS: outra classe de sons. 


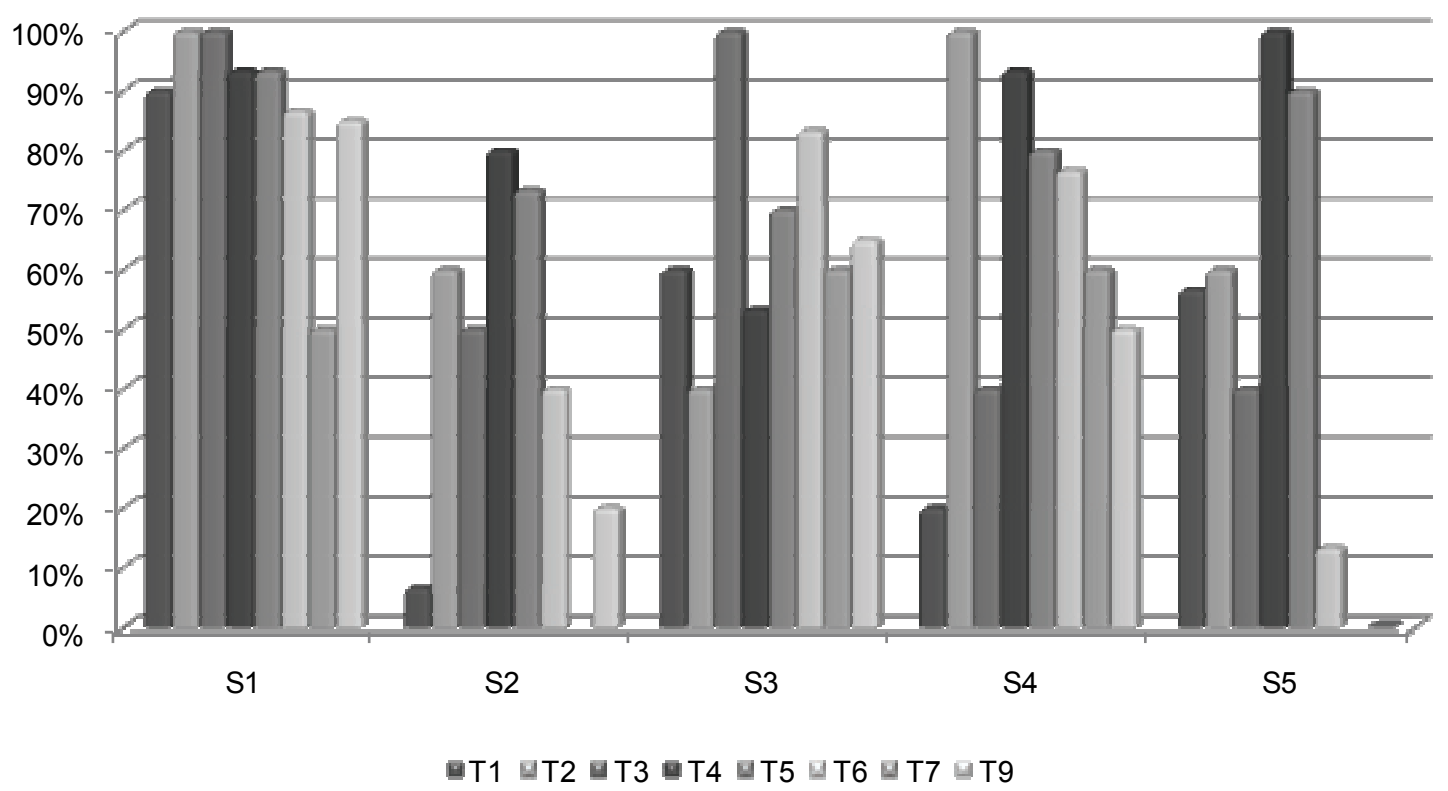

Legenda: T1 - segmentação de frases em palavras; T2 - realismo nominal; T3 - detecção de rimas; T4 - síntese silábica; T5 - segmentação silábica; T6 - detecção de sílabas; T7 - reversão silábica; T8 - exclusão fonêmica; T9 - detecção de fonemas; T10 - síntese fonêmica; T11 - segmentação fonêmica; T12 - reversão fonêmica.

\section{Figura 1 - Desempenho de cada um dos sujeitos em tarefas de consciência fonológica}

\section{DISCUSSÃO}

Quanto ao inventário fonético, apenas dois dos sujeitos (S4 e S5) apresentavam alteração no pré-tratamento, sendo que a maioria desses sons foi adquirida após a terapia, permanecendo um ausente. Alguns estudos ${ }^{16,19}$ referiram o acréscimo de sons no inventário fonético após tratamento com base fonológica.

No que se refere ao inventário fonológico, o S1 acrescentou o único fonema ausente na avaliação inicial; o S2 acrescentou apenas um dos seus dois fonemas ausentes e o outro tornou-se parcialmente adquirido; e o S3 não adquiriu o seu único fonema ausente. Já o S4 e o S5, não apresentaram aquisição de nenhum fonema, apenas alguns se tornaram parcialmente adquiridos em seus inventários fonológicos, após serem submetidos a terapia com base fonológica. Observou-se um aumento no percentual de produções corretas dos fonemas no inventário fonológico para todos os sujeitos, embora não o suficiente para eliminar todas as substituições ou apagamentos que ocorriam.

Quanto aos tipos de generalizações pesquisadas, observa-se que os cinco sujeitos da pesquisa apresentaram generalizações, alguns mais outros menos de acordo com suas possibilidades de ocorrência. A generalização é usada em várias pesquisas ${ }^{16-18,20-22}$ para verificar a evolução terapêutica de sujeitos com desvio fonológico, pois ao se obter generalizações a terapia torna-se mais rápida, não precisando ensinar todos os fonemas alterados na fala da criança em todas as posições que ocorrem.

Nas tarefas de consciência fonológica, o S1 foi o único que obteve êxito em todas as tarefas analisadas. De acordo com algumas pesquisas, habilidades em consciência fonológica pode ser um fator favorecedor na terapia para os desvios fonológicos ${ }^{23,24}$.

Assim como a terapia fonológica contribui de maneira positiva para o desenvolvimento de habilidades em consciência fonológica ${ }^{7}$, utilizar atividades que envolvam esta habilidade pode levar a uma mudança no sistema fonológico da criança ${ }^{23,24}$. O S1 apresentou um bom resultado nas habilidades de consciência fonológica e adquiriu sons nos inventários fonético e fonológico, fato este referido por um estudo ${ }^{25}$ em que afirma ser possível que as crianças que tem boas habilidades de consciência fonológica respondem melhor terapia da fala.

O S1 foi o que obteve maiores percentuais de generalização a itens lexicais não utilizados no tratamento e para outra posição na palavra. Esses dois tipos de generalização também foram observados em outros estudos ${ }^{11,17,18}$. Pesquisas revelam que a aquisição dos inventários fonético e fonológico, bem como as generalizações obtidas, tem como motivador a seleção dos alvos de tratamento ${ }^{16-18}$. 
O S2 apresentou um desempenho inferior em consciência fonológica em relação aos outros sujeitos analisados e foi o que menos evoluiu na terapia. O único som ausente no pré-tratamento não foi adquirido e nem generalizado quando possível. Tal fato vai ao encontro de pesquisas que demonstraram que crianças com alterações fonológicas podem ter um baixo desempenho nas habilidades em consciência fonológica ${ }^{3,5,23}$.

O S3 obteve êxito em doze, de dezesseis subtarefas de consciência fonológica, porém, na evolução da terapia não se detectou aquisições no inventário fonológico e um percentual baixo quanto aos tipos de generalizações possíveis.

O S4 foi o segundo sujeito que mais evoluiu na terapia acrescentando oito sons no inventário fonético e apresentando generalizações para todos os tipos pesquisados. Este sujeito obteve um bom resultado em consciência fonológica com êxito em treze das dezesseis subtarefas aplicadas. Pesquisas ${ }^{12,17,18}$ referem ter observado aquisições de sons nos inventários fonológicos após a intervenção com base fonológica.

O S5 apresentou êxito em dez, de dezesseis subtarefas de consciência fonológica e teve boa evolução na terapia, apresentado melhoras nos inventários fonético e fonológico e nos tipos de generalização. A generalização dentro de uma classe de sons e para outra classe de sons são especialmente desejadas por contribuírem com mudanças mais globais no inventário fonológico ${ }^{11}$.

Ao analisar o desempenho em consciência fonológica e a terapia fonológica, quem mostrou melhores resultados nesta avaliação também apresentou maiores evoluções no tratamento (S1). Todavia, o S3 apresentou um bom resultado nas tarefas de consciência fonológica, comparado aos outros sujeitos, e não apresentou muitas melhoras no inventário fonológico e nem quanto aos tipos de generalização.

Ainda que não se tenha avaliado a consciência fonológica pós-terapia, os resultados observados neste estudo ventilam a hipótese de que pode haver uma relação entre tal habilidade e a terapia para os desvios fonológicos. Acredita-se que a terapia fonológica pode influenciar de maneira positiva no desenvolvimento das habilidades em consciência fonológica ${ }^{7}$ e esta, na terapia para os desvios fonológicos.

Um estudo ${ }^{26}$ aponta para a necessidade de avaliar as habilidades em consciência fonológica de todas as crianças com desvio fonológico, talvez com especial atenção para aquelas que exibem numerosos erros atípicos de sons, pois estas crianças podem estar em risco para o desenvolvimento de habilidades em consciência fonológica e consequentemente para a alfabetização. Este mesmo estudo refere que várias crianças com desvio fonológico executaram muito bem as tarefas de consciência fonológica, apresentando relativamente poucos erros atípicos, ao contrário das crianças que não foram bem na realização das tarefas de consciência fonológica. O desenvolvimento adequado do sistema fonológico é a base para o desenvolvimento posterior de outras capacidades linguísticas, isto ressalta a importância de pesquisar uma terapia fonológica com ênfase no processamento fonológico ${ }^{5}$.

Atualmente, se tem poucas pesquisas a respeito da interferência da consciência fonológica na evolução da terapia fonológica. Os resultados demonstrados neste estudo baseiam-se na fala de poucos sujeitos, portanto, seria importante que outros estudos fossem realizados no intuito de investigar essa possível relação. Acredita-se que o entendimento dessas relações possa nortear de maneira mais eficaz o tratamento de crianças com desvio fonológico.

\section{CONCLUSÕES}

Observa-se que, para os sujeitos desta pesquisa, não houve, em sua totalidade, relação entre as habilidades em consciência fonológica e o progresso na terapia fonológica.

A literatura aponta que crianças com desvio fonológico podem ser capazes de responder adequadamente a tarefas metalinguísticas como a consciência fonológica, sem que essa condição as auxilie a corrigir os desvios de sua fala. Nesse estudo foram verificadas evoluções quanto aos inventários fonético e fonológico e aos tipos de generalização, no entanto, tais evoluções não estiveram relacionadas com a habilidade metalinguística investigada.

Considerando que os dados analisados foram de apenas cinco sujeitos, sugere-se que outros estudos sejam realizados contando com uma amostra maior. Além disso, sugere-se que a consciência fonológica seja reavaliada na alta terapêutica. 


\section{ABSTRACT}

This study aimed to analyze the phonological awareness skills and progress (phonetic and phonological inventory and generalizations) in phonological therapy. The research group consisted of five children presenting speech disorders, aged 5:0 and 6:11, subjected to phonological therapy. The results of the phonological awareness assessment were analyzed before the treatment in order to verify the performance of children and their phonological knowledge. The evaluation was performed before and after the treatment. It was possible to obtain the phonetic and phonological inventories of the subjects and, after that, the generalizations obtained with phonological therapy were analyzed (considering lexical items that were not used during treatment, other positions in word, within one sound class or another class of sounds). The results showed that there is no relation between the performance in phonological awareness tasks and the evolution of the phonological therapy. On the other hand, the results agree with the research findings from the literature, reinforcing the idea that, children with phonological disorder can be able to react properly to metalinguistic tasks such as phonological awareness without being able to correct their speech problems. Finally, it can be conclude that this research subject should be investigated deeper and another analysis should be carried out involving a higher number of subjects, including the phonological awareness evaluation before and after therapy. By doing this, it will be possible to obtain other important data to improve the treatment of phonological disorder.

KEYWORDS: Speech; Speech Disorders; Speech Perception; Speech Therapy; Generalization, Response

\section{REFERÊNCIAS}

1. Cielo CA. Habilidades em consciência Fonológica em crianças de 4 a 8 anos de idade. Pró-Fono. 2002; 14(3):301-12.

2. Rvachew S, Grawburg M. Correlates of Phonological Awareness in Preschoolers With Speech Sound Disorders. J Speech Lang Hear Res. 2006; 49:74-87.

3. Morales MV, Mota HB, Keske-Soares M. Consciência fonológica: desempenho de crianças com e sem desvios fonológicos evolutivos. Pró-Fono. 2002; 14(2):153-64.

4. Holm A, Farrier F, Dodd B. Phonological awareness, reading accuracy and spelling ability of children with inconsistent phonological disorder. Int J Lang Comm Dis. 2008;43(3):300-22.

5. Mota H, Melo Filha MGC. Habilidades em consciência fonológica de sujeitos após realização de terapia fonológica. Pró-Fono. 2009;21(2):119-24.

6. Marchetti PT, Mezzomo CL, Cielo Desempenho em consciência silábica e fonêmica em crianças com desenvolvimento de fala normal e desviante. Rev. CEFAC. 2010;12(1):12-20.

7. Marchetti PT, Mezzomo CL, Cielo CA. Habilidades em consciência silábica e fonêmica de crianças com fala desviante com e sem intervenção fonoaudiológica. Pró-Fono. 2010;5(1):80-7.
8. Souza TNU, Avila CRB. Gravidade do transtorno fonológico, consciência fonológica e praxia articulatória em pré-escolares. 2011. Rev Soc Bras Fonoaudiol. 2011;16(2):182-8.

9. Donicht G, Nazari GT. Relação entre desvios fonológicos e consciência fonológica. Letrônica. 2010;3(1):22-41.

10. Rizzon GF, Chiechelski P, Gomes E. Relação entre consciência fonológica e desvio fonológico em crianças da $1^{\underline{a}}$ série do ensino fundamental. Revista CEFAC. 2009;11(sup 2):201-7.

11. Gierut JA. Complexity in phonological treatment: clinical factors. Lang, Speech Hear Serv Schools. 2001; 32:229-41.

12. Mota HB, Bagetti T, Keske-Soares M, Pereira LF. A generalização baseada nas relações implicacionais em sujeitos submetidos à terapia fonológica. Pró-Fono. 2005;17:99-110.

13. Tyler A, Figursky GR. Phonetic inventory chances after treating distinctions along implicatonal hierarchy. Clin Linguist Phon. 1994;8(2):91-107.

14. Weiner F. Treatment of phonological disability using the method of meaningful minimal contrast: two case studies. J Speech Hear Dis.1981;46:97-103.

15. Gierut JA. The conditions and course of clinicallyinduced phonological change. J Speech Hear Res. Bloomington.1992;35:1049-63. 
16. Williams AL. Assessment, target selection, and intervention: dynamic interactions within a systemic perspective. Top Lang Dis. 2005;25(3):231-42.

17. Ceron MI, Keske-Soares M. Terapia Fonológica: a generalização a itens não utilizados no tratamento (outras palavras). Revista CEFAC. 2007;9(4):453-60.

18. Ceron MI, Keske-Soares M. Terapia Fonológica: a generalização dentro de uma classe de sons e para outras classes de sons. Revista CEFAC.2008;10(3):311-20.

19. Ceron MI, Keske-Soares M, Gonçalves GF. Escolha dos sons-alvo para terapia: análise com enfoque em traços distintivos. Rev Soc Bras Fonoaudiol. 2010; 15(2):270-6.

20. Gierut JA, Dale RA. Comparability of Lexical Corpora: Word frequency in phonological generalization. Clin Linguist Phon. 2007;21(6):423-33.

21. Ceron MI, Keske-Soares M. Terapia Fonológica: a generalização para outra posição na palavra. Revista CEFAC. 2009;11(2):199-206.
22. Cummings $A E$, Barlow JA. A comparison of word lexicality in the treatment of speech sound disorders. Clin Linguist Phon. 2011;25(4):265-86. 23. Laing SP, Espeland W. Low intensity phonological awareness training in a school classroom for children with communication impairments. J Commun Dis. 2005;38(1):65-82.

24. Spíndola RA, Payão LMC, Bandini HHM. Abordagem fonoaudiológica em desvios fonológicos fundamentada na hierarquia dos traços distintivos e na consciência fonológica. Revista CEFAC.2007;9(2):180-9.

25. Rvachew S, Chiang PY, Evans N. Characteristics of speech errors produced by children with and without delayed phonological awareness skills. Lang, Speech Hear Serv Schools. 2007;38:60-71.

26. Preston J, Edwards ML. Phonological Awareness and Types of Sound Errors in Preschoolers with Speech Sound Disorders. J Speech Lang Hear Res.2010; 53(1):44-60.
http://dx.doi.org/10.1590/S1516-18462012005000093

Recebido em: 25/08/2011

Aceito em: 09/02/2012

Endereço para correspondência:

Roberta Feitas Dias

Rua Antero Corrêa de Barros, 244 - Centro

Santa Maria - RS

CEP: 97010-120

E-mail: robertafdias@ hotmail.com 